\title{
Unexpected Complications after Corrective Spinal Fusion Surgery for Adult Spinal Deformity with Severe Hip Contracture
}

\section{Yasushi Yoshikawa ${ }^{1}$, Mizuki Toura ${ }^{1}{ }^{12}$, Yoshifumi Kudo ${ }^{1}$, Ichiro Okano ${ }^{1}$, Masanori Nishi ${ }^{1}$, Tomoaki Toyone ${ }^{1}$, Katsunori Inagaki ${ }^{1}$}

Learning Point of the Article:

Pre-operative hip evaluation and careful indication before spinal fusion surgeries are recommended for a successful surgery.

\section{Abstract}

Introduction: In cases with markedly decreased hip function, patients predominantly utilize spine movement while standing up to compensate for the hip malfunction. However, spinal fusion surgeries might lead to the disruption of this compensatory mechanism, resulting in difficulties in walking and standing up as well as proximal junctional failure (PJF) due to the excessive stress on the spine caused by the pendulum-like motion needed for standing up. Hence, in patients with severe hip pathology, surgeons should be cautious about the indication for spinal fusion, which inevitably affects spinal mobility. This is the first report presenting a case that supports the aforementioned theory.

Case Report: In this study, we report the case of a 76-year-old Japanese woman who underwent corrective spinal fusion surgery for spinal scoliosis secondary to hip contracture. The patient exhibited post-operative complications, such as unexpected difficulty in walking and standing up and PJF. The patient underwent a revision spinal surgery with an extension of spinal fusion for PJF and muscle release around the hip for hip contracture which resulted in improved walking and standing movements with no reports of pain.

Conclusion: Spinal fusion surgeries performed on patients with severe hip pathology could cause early PJFs and unexpected decline in activities of daily living. Patients with such risks often do not complain of hip symptoms before spinal correction surgery. Surgeons should routinely evaluate hip joints and be cautious about the indication for spinal fusion which inevitably affects spinal mobility.

Keywords: Hip joint contracture, spinal correction surgery, proximal junctional failure, muscle release around the hip.

\section{Introduction}

Offierski and Macnab first introduced the concept of hip-spine syndrome in 1983 [1]. The hip and vertebral pelvic girdle are adjacent structures, and pathological conditions in one anatomical region affect the function of the part causing pathological conditions in that region $[1,2,3,4,5,6]$. In recent years, spinal alignment correction with spinal fusion has been commonly performed for adult spinal deformity and has exhibited an improvement regarding pain and activities of daily living (ADLs). However, these spinal correction procedures may cause unsatisfactory results in patients with a limited hip range of motion (ROM). In these patients, the spinal motion is likely to compensate for the limited hip motion. Therefore, the immobilization of spinopelvic region by fusion surgery may cause loss of compensation and problems which affect the patient's ADLs $[7,8]$. In this report, we present a case of longterm severe hip contracture and a proximal junctional failure (PJF) in the early post-operative period after corrective spinopelvic fixation for deformity secondary to hip contracture.

\section{Case Report}

A 76-year-old woman previously diagnosed with tuberculous arthritis in the right hip visited our spine care center for the progression of scoliosis and a right-sided abdominal pain, which
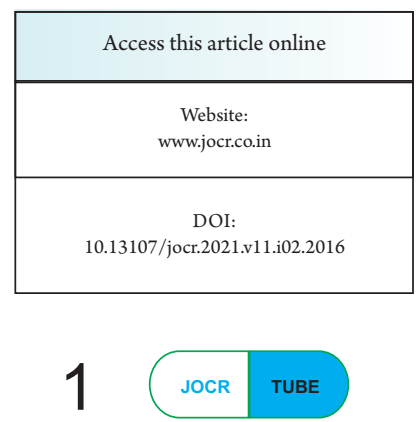

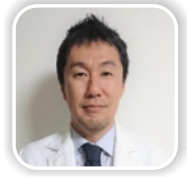

Dr. Yasushi Yoshikawa

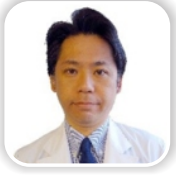

Dr. Yoshifumi Kudo Author's Photo Gallery

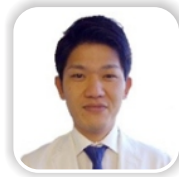

Dr. Masanori Nish

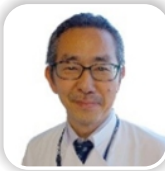

Dr. Tomoaki Toyone

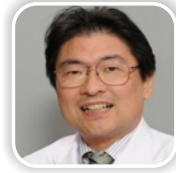

Dr. Katsunori Inagaki

'Department of Orthopaedic Surgery, Showa University School of Medicine, Shinagawa, Tokyo, Japan, Department of Orthopaedic Surgery, Tokyo Kyosai Hospital, Meguro, Tokyo, Japan.

Address of Correspondence

Dr. Yasushi Yoshikawa,

Department of Orthopaedic Surgery, Showa University School of Medicine, Shinagawa, Tokyo, Japan

E-mail: yoshikawa@med.showa-u.ac.jp

Journal of Orthopaedic Case Reports | pISSN 2250-0685 | eISSN 2321-3817 | Available on www.jocr.co.in | doi:10.13107/jocr.2021.v11.i02.2016 This is an Open Access article distributed under the terms of the Creative Commons Attribution Non-Commercial License (http://creativecommons.org/licenses/by-nc/3.0) which permits unrestricted non-commercial use, distribution, and reproduction in any medium, provided the original work is properly cited. 


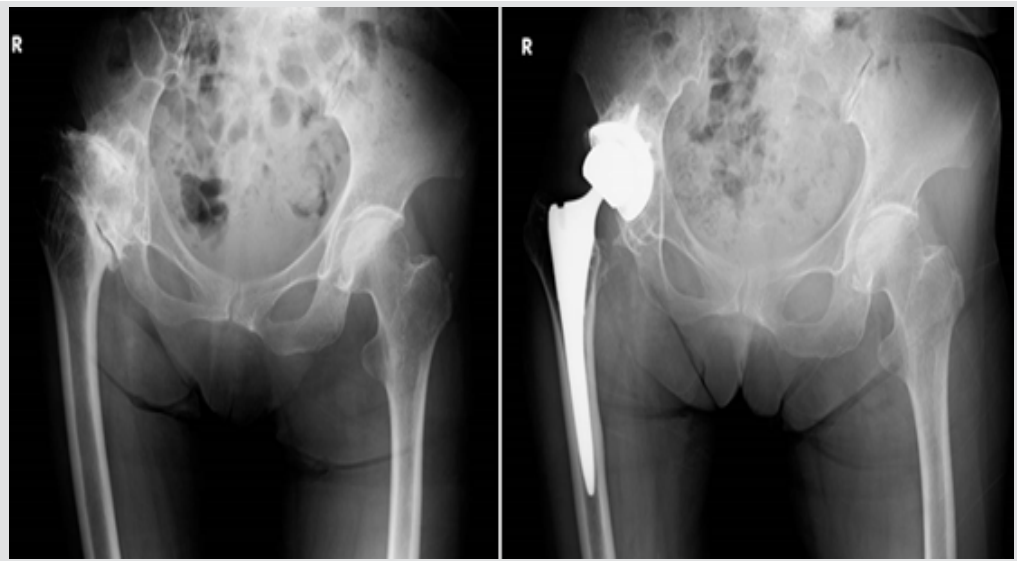

Figure 1: Pre- and post-arthroplasty anteroposterior radiographs of the bilateral hip joints. (a) Radiograph showing an osteoarthritis of the right hip after tuberculous arthritis, a shortening of the right lower limb, an adduction contracture of the right hip, and pelvic tilting. (b) Post-operative radiograph showing a total hip arthroplasty in the right hip. Leglength discrepancy, adduction contracture, and pelvic tilting persisted.

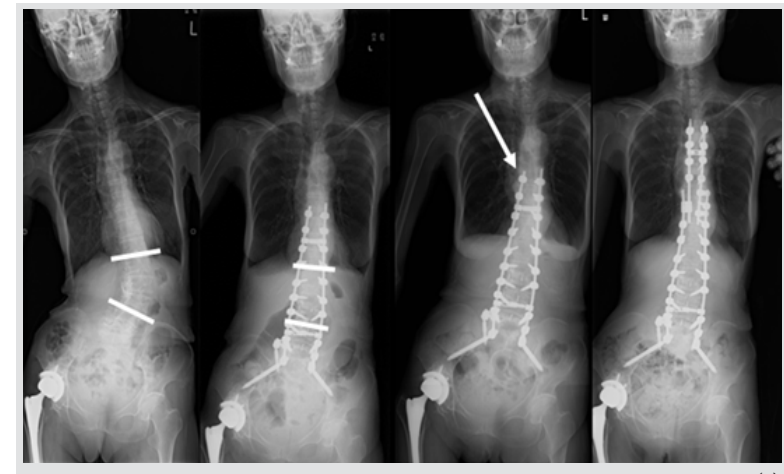

Figure 2: Anteroposterior radiographs of the whole-spine standing radiographs. (a) Pre-operative radiograph showing coronal imbalance with a lumbar left convex scoliosis Cobb angle of $35^{\circ}$ and $35 \mathrm{~mm}$ rightward shift of the $\mathrm{C} 7$ central sacral vertical line (CSVL). (b) Post-operative radiograph after a combined anterior and posterior corrective spinopelvic fixation from $\mathrm{T} 9$ to the pelvis illustrating an improvement in the lumbar left convex scoliosis Cobb angle to $12^{\circ}$; however, the C7 CSVL was overshifted by $48 \mathrm{~mm}$ to the left. (c) Six weeks after the spine surgery, T9 collapsed (arrow), and (d) the fusion was extended to T5. was caused by an impingement between a rib and the iliac crest. The patient had undergone a total hip arthroplasty (THA) for the right hip pain, right hip abduction contracture, and right leg shortening 6 years prior. After THA, the patient had been pain free; however, the right lower limb shortening and right hip abduction contracture persisted (Fig. 1a, b). At the time of the initial examination for spinal deformity, she could walk and stand up with a single cane. The spinal alignment parameters indicated a severe coronal imbalance with $35^{\circ}$ lumbar left convexity Cobb angle and 35 mmrightward shifted C7 central sacral vertical line (CSVL) (Fig. 2a). In contrast, sagittal balance parameters were relatively preserved on the standing wholespine radiographs with no significant mismatch in the SRSSchwab classification; lumbar lordosis (LL) angle was $43^{\circ}$, and pelvic incidence (PI) minus LL angle was $5^{\circ}$ (Fig. 3a) $[9,10]$. The ROM of the right hip showed a markedly limited flexion to $50^{\circ}$ as well as an adduction contracture. For the spinal deformity, a combined anterior and posterior corrective fusion from T9 to the iliac bone was performed. Postoperatively, the coronal imbalance improved to a Cobb angle of $12^{\circ}$; however, post-operative C7CSVL was shifted $48 \mathrm{~mm}$ to the left (Fig. 2b).

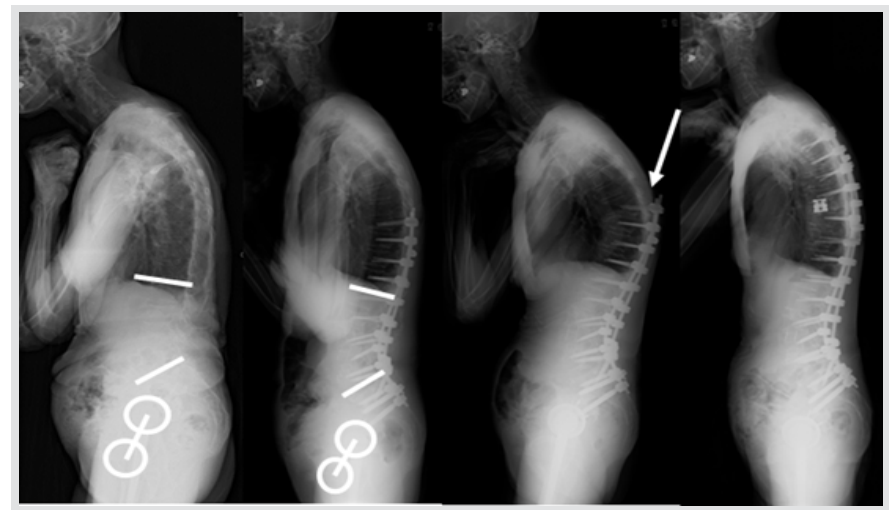

Figure 3: Lateral whole-spine standing radiographs. (a) Pre-operative radiograph showing a lumbar lordosis (LL) angle of $43^{\circ}$ and pelvic incidence (PI) minus LL angle of $5^{\circ}$. (b) Postoperative radiograph after the spinopelvic fixation showing an LL angle of $42^{\circ}$ and a PI-LL angle of $5^{\circ}$. (c) Six weeks after the spine surgery, T9 collapsed (arrow), and (d) the fusion was extended to T5.
The sagittal alignment was unchanged (Fig. 3b). These coronal parameter changes resulted in an improvement in the rightsided abdominal pain due to rib iliac impingement. However, it similarly proved difficult to keep sitting as well as stand up (Video 1) and walk due to a leftward overshift of the trunk (Fig. $4 \mathrm{a}, \mathrm{b}, \mathrm{c})$. In addition, 1 month postoperatively, $\mathrm{T} 9$ appeared to have collapsed and required a revision spinal surgery with an upward extension of fusion to T5 (Fig. 2c, d and 3c, d). Since walking and standing difficulties persisted, muscle release around the hip was additionally performed to improve the ROM of the right hip. The iliopsoas, gluteus maximus, and adductor muscle tendons were dissected. The right hip ROM improved to $75^{\circ}$ of flexion after muscle release. The patient returned home after a 3 -month rehabilitation with an improvement in walking and standing movements with no pain; however, she has not attained her pre-operative level of function.

\section{Discussion}

Spinopelvic corrective fixation is a common procedure for adult
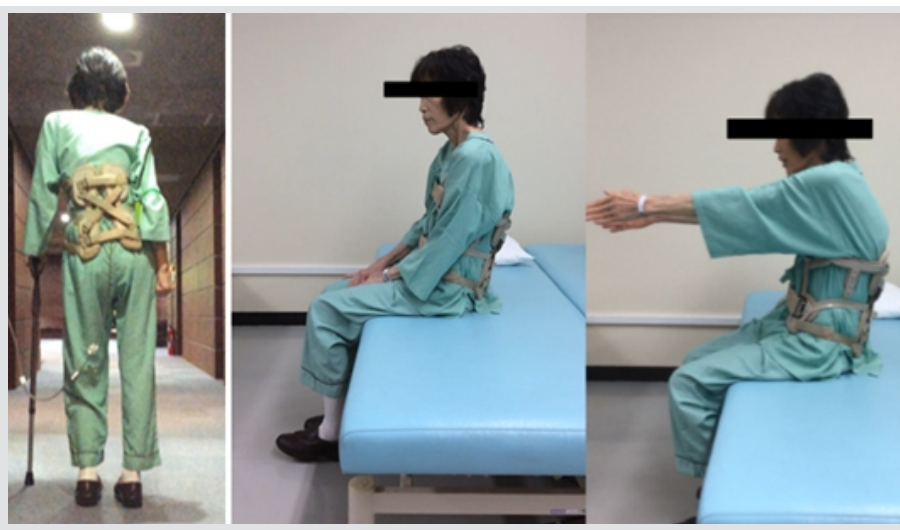

Figure 4: Post-operative photographs during walking and standing-up exercises after spinal fusion. (a) During walking, the trunk is tilted to the left. (b) The trunk is leaned back while sitting. (c) Inability to shift the center of gravity forward when standing up, and the patient could not stand up without support. 


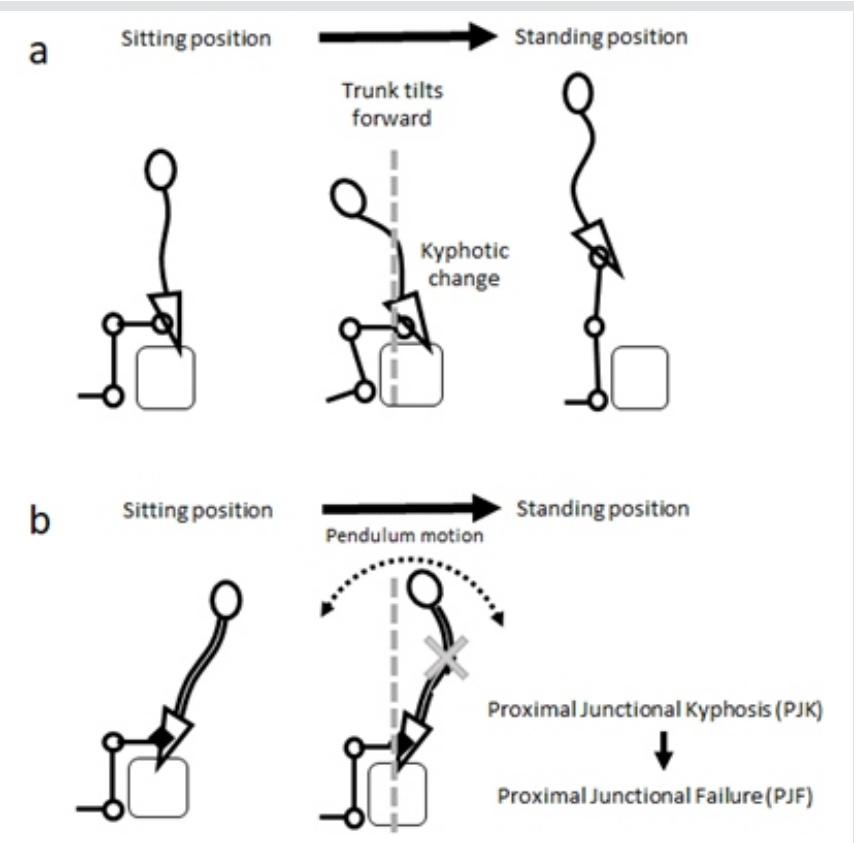

Figure 5: Schematic images of the center of gravity shift during standing-up motion. (a) Normal center of gravity shift; the hip flexion and spinal kyphotic movement move the center of gravity forward. (b) Center of gravity shift in a patient with post-spinal fusion status and hip contracture. Since hip flexion and spinal kyphotic movement are impossible, the patients should use pendulum-like movements to shift the center of gravity anteriorly. This may contribute to the early onset of proximal junctional failure.

spine deformity with ADL decline. Katayanagi et al. noted that $32 \%$ of patients who underwent long spinal fusion for adult spinal deformity demonstrated improved stand-up motion after surgery [11]. In contrast, one disadvantage of spinal fusion surgery was that the fusion inevitably sacrificed the mobility of the spine [12]. Morimoto et al. reported that abduction and adduction contractures of the hip joint caused lateral pelvic tilting and lumbar scoliosis. Lumbar scoliosis in this patient likely occurred to compensate for the lateral pelvic tilting due to leg length discrepancy and adduction contracture of the hip joint secondary to tuberculous arthritis in childhood [13].

In this case, the alleviation of pain caused by rib iliac impingement associated with scoliosis was achieved with spinal coronal plane correction surgery. However, because of pelvic tilting due to residual hip joint pathology, this correction likely caused the coronal imbalance to the other side and deterioration of standing-up and walking functions probably due to limited spine motion. During the standing-up motion, anterior movement of the center of gravity is necessary to complete the motion, and hip flexion, decreased LL, and increased thoracic kyphosis contributed to this center of gravity shift [12]. In this patient, spinal movements played the main role in this gravity center shift because of limited hip flexion [14]. This compensatory mechanism probably became dysfunctional after the long spinopelvic fixation procedure. The patient required a pendulum-like motion of the trunk to shift the center of gravity forward, and the repetitive stress on the spine by this pendulum-like motion might have caused the
PJF in the early post-operative period (Fig. 5a, b).

Previously, Riviere et al. investigated the interaction between the hip and spine while standing and sitting and reported the concept of hip and spine users. Hip users primarily utilize the hip joint movement to stand up, whereas spine users utilize the spine movement more dominantly while standing up [15]. It is expected that spine fusion surgery will have a more significant negative impact on stand-up motion in spine users than in hip users. Supposedly, this patient was more likely a spine user because she demonstrated long-term hip ROM restriction due to tuberculous arthritis. When spinal fixation is indicated in a spine user, we suggest that hip pathology should be addressed first or at least simultaneously to avoid unexpected ADL declines.

In this case, we performed dissections of the gluteus maximus tendon to improve hip flexion and the abductor muscles and iliopsoas tendons to improve hip abduction. These procedures were effective in improving the patient's standing-up and walking abilities. Moreover, these muscle releases should be considered as salvage surgeries for patients with hip contracture and new-onset standing/walking difficulties after spinal deformity corrections. However, soft-tissue surgery alone might not be adequate to improve function in some cases, such as in patients with severe supporting muscle atrophy, and cannot be applied in all cases. Therefore, a pre-operative discussion between hip and spine surgeons is warranted to improve the outcomes of patients with hip contracture and spine deformity and avoid such complications.

\section{Conclusion}

Spinal fusion surgeries performed on patients with severe hip pathology could cause early PJFs and unexpected decline in ADL. Patients with such risks often do not complain of hip symptoms before spinal correction surgery. Surgeons should routinely evaluate hip joints and be cautious about the indication for spinal fusion which inevitably affects spinal mobility.

\section{Clinical Message}

Spinal correction surgery is an effective procedure for patients with a severe decline in ADL. Pre-operative hip evaluation and careful indication are recommended for a successful surgery. 


\section{References}

1. Offierski CM, MacNab I. Hip-spine syndrome. Spine 1983;8:316-21.

2. Roussouly P, Pinheiro-Franco JL. Biomechanical analysis of the spino-pelvic organization and adaptation in pathology. Eur Spine J 2011;20:609-18.

3. Jackson RP, McManus AC. Radiographic analysis of sagittal plane alignment and balance in standing volunteers and patients with low back pain matched for age, sex, and size. A prospective controlled clinical study. Spine 1994;19:16118.

4. Fogel GR, Esses SI. Hip spine syndrome: Management of coexisting radiculopathy and arthritis of the lower extremity. Spine J 2003;3:238-41.

5. Radcliff KE, Orozco F, Molby N, Delasotta L, Chen E, Post Z, Ong A. Change in spinal alignment after total hip arthroplasty. Orthop Surg 2013;5:261-5.

6. Ben-Galim P, Ben-Galim T, Rand N, Haim A, Hipp J, Dekel S, Floman Y. Hip-spine syndrome: The effect of total hip replacement surgery on low back pain in severe osteoarthritis of the hip. Spine 2007;32:2099-102.

7. Buckland AJ, Puvanesarajah V, Vigdorchik J, Schwarzkopf R, Jain A, Klineberg EO, et al. Dislocation of primary total hip arthroplasty is more common in patients with a lumbar spinal fusion. Bone Joint J 2017;99-B:585-91.

8. An VV, Phan K, Sivakumar BS, Mobbs RJ, Bruce WJ. Prior lumbar spine fusion is associated with an increased risk of dislocation and revision in total hip arthroplasty: A metaanalysis. J Arthroplasty 2018;33:297-300.

Conflict of Interest: Nil Source of Support: Nil

Consent: The authors confirm that informed consent was obtained from the patient for publication of this case report
9. Schwab F, Ungar B, Blondel B, Buchowski J, Coe J, Deinlein D. Scoliosis research society-schwab adult spinal deformity classification. Spine 2012;37:1077-82.

10. Schwab F, Blondel B, Bess S, Hostin R, Shaffrey C, Smith J. Radiographical spinopelvic parameters and disability in the setting of adult spinal deformity. Spine 2013;38:80312.

11. Katayanagi J, Iida T, Hayamizu A, Matsumoto K, Ohyama Y, Mine $\mathrm{K}$, et al. Effect of long spinal fusion including the pelvis on activities of daily living related to lumbar spinal function in adults with spinal deformity. J Orthop Sci 2007;12:526-32.

12. Furuhashi H, Togawa D, Koyama H, Hoshino H, Yasuda T, Matsuyama Y. Repeated posterior dislocation of total hip arthroplasty after spinal corrective long fusion with pelvic fixation. Eur Spine J 2017;26:100-6.

13. Morimoto T, Sonohata M, Kitajima T, Yoshihara T, Hirata H, Mawatari M. Hip-spine syndrome: The coronal alignment of the lumbar spine and pelvis in patients with ankylosed hips. Spine Surg Relat Res 2020;4:37-42.

14. Buckland AJ, Steinmetz L, Zhou P, Vasquez-Montes D, Kingery M, Stekas ND. Spinopelvic compensatory mechanisms for reduced hip motion (ROM) in the setting of hip osteoarthritis. Spine Deform 2019;7:923-8.

15. Riviere C, Lazennec JY, Van Der Straeten C, Auvinet E, Cobb J, Muirhead-Allwood S. The influence of spine-hip relations on total hip replacement: A systematic review. Orthop Traumatol Surg Res 2017;103:559-68.

\section{How to Cite this Article}

Yoshikawa Y, Toura M, Kudo Y, Okano I, Nishi M, Toyone T, Inagaki K. Unexpected Complications after Corrective Spinal Fusion Surgery for Adult Spinal Deformity with Severe Hip Contracture. Journal of Orthopaedic Case Reports 2021 February; 11(2):37-40. 